\title{
Late recurrent cutaneous breast angiosarcoma in an elderly woman: A case report
}

\author{
FAUSTO FAMA', LUANA LICATA, ALESSANDRA VILLARI, \\ JESSICA PALELLA, GIUSEPPE SPECIALE and MARIA GIOFFRE'-FLORIO \\ Department of Human Pathology, University Hospital of Messina, Messina 98125, Italy
}

Received March 9, 2015; Accepted January 12, 2016

DOI: $10.3892 / \mathrm{ol} .2016 .4389$

\begin{abstract}
Breast angiosarcomas are malignant tumours of the vascular endothelium that arise frequently following radiation therapy. Their clinical and radiological aspects are highly heterogeneous. The current study reports an unusual case, never previously reported, of a late recurrent breast angiosarcoma occurring in an 83-year old female patient 11 years after a breast-conserving surgery followed by radiation therapy for an invasive ductal carcinoma, and 5 years after her initial angiosarcoma excision. The first physician to examine the patient noted a palpable mass near the scar and, following ultrasonography, described the breast lesion as suggestive of an abscess, despite the previous history of neoplasia. Typically, recurrences of breast angiosarcoma occur within the first postsurgical year. The present patient remains alive at 25 months after her last surgical treatment, and no evidence of any local or distant disease is observable.
\end{abstract}

\section{Introduction}

Breast angiosarcoma (BA) is a rare malignant endothelial tumour $(1,2)$. This tumour may be distinguished into primary and secondary forms; the overall yearly incidences are reported in the literature as $0.002-0.05 \%$ and $0.01-0.02 \%$, respectively (1). The aetiology of primary BA, typically occurring in women $<40$ years of age, remains unknown (2). These neoplasms present as a growing, palpable mass that arises in the non-irradiated breast parenchyma, with rare skin involvement (3). Secondary BA may occur more frequently after the fourth decade of life, particularly in patients who have already been treated with radiation therapy following breast-conserving surgery (2-5). It presents as painless, multifocal skin changes that may be neglected or misdiagnosed, or as a breast lump (3). Postoperative chronic lymphoedema is also indicated as a

Correspondence to: Professor Fausto Fama', Department of Human Pathology, University Hospital of Messina, 1 Via Consolare Valeria, Messina 98125, Italy

E-mail: famafausto@yahoo.it

Key words: breast, angiosarcoma, radiotherapy, recurrence potential causal factor for the development of angiosarcoma in the breast and in the upper extremities, where it is known as Stewart-Treves syndrome (2). BA generally arises 3-12 years after the initial surgical and radiation therapies (6). Recurrences are rare $>2$ years after surgery, and their predominant characteristics are clinical and imaging heterogeneity $(1,5)$.

The current study presents an unusual case of non-metastatic late recurrent BA in an elderly woman with atypical clinical features, including a long time interval between the first and second occurrences of angiosarcoma, and a 25-month disease-free survival period.

\section{Case report}

An 83-year-old female, exhibiting a superficial, palpable painless lump in the left breast that had appeared $\sim 1$ month prior, was admitted to the General Surgery Unit of the University Hospital of Messina (Messina, Italy) in January 2013. Her history included hypertension, gastritis and osteoarthritis.

In 2002, the patient had undergone a left superoexternal quadrantectomy with synchronous ipsilateral axillary lymph node dissection for an invasive ductal carcinoma. The lymph nodes did not appear to be involved despite the presence of palpable nodes in the axilla, and the patient also developed slight postoperative lymphoedema. She was then assigned to receive adjuvant radiotherapy in association with a 5-year hormone-therapy schedule, consisting of tamoxifen ( $20 \mathrm{mg} /$ day). Whole breast irradiation was performed at a dose of $50 \mathrm{~Gy}$ in 25 fractions (200 cGy/fraction), plus a boost of 10 Gy (200 cGy/fraction) on the breast bed, between May and June of 2002.

In 2008, the patient underwent a Madden mastectomy for a secondary angiosarcoma (7). The anatomopathological examination of the latter indicated a cutaneous, well-differentiated angiosarcoma of $15 \mathrm{~mm}$ in its largest diameter, with moderately atypical spindle cells delimiting thin vascular spaces; the tissue also exhibited frequent mitoses, a moderate (30\%) MIB-1 labelling index (LI), and an intense immunoreaction to endothelial markers, including cluster of differentiation (CD)31 and CD34. No adjuvant treatment was performed following the last surgical procedure, in accordance with the recommendation of the International Society of Geriatric Oncology (8).

When assessed in January 2013, the patient presented a cutaneous mass in the upper-inner quadrant of the breast, 


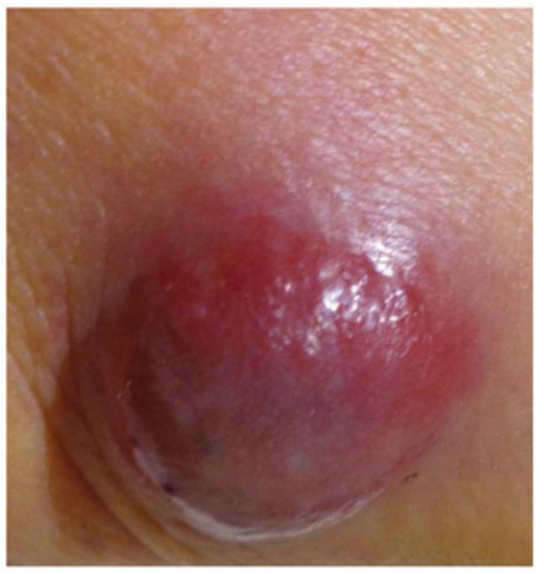

Figure 1. Cutaneous painless lump in the superomedial quadrant of the left breast surrounded by a hyperaemic area

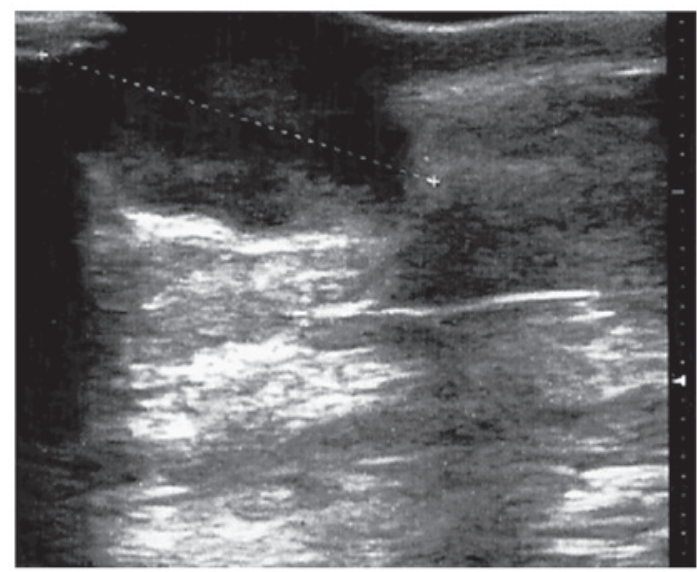

Figure 2. Hypo-anechoic oval nodule (22 $\mathrm{mm}$ in largest diameter) with posterior acoustic enhancement.

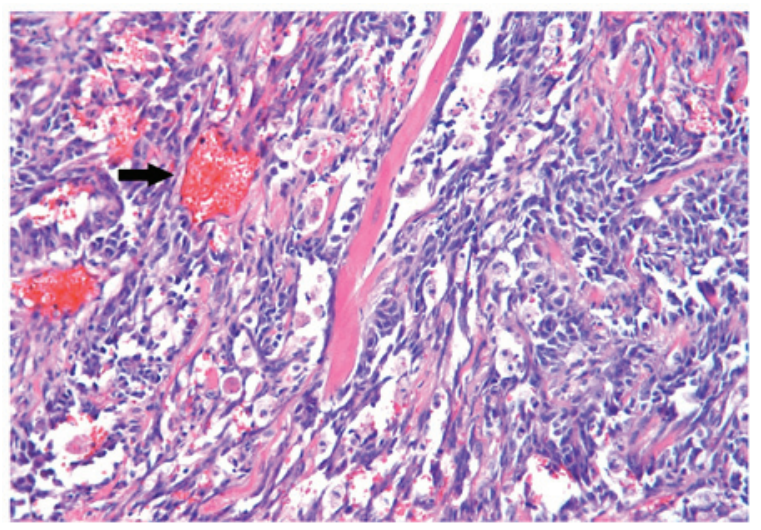

Figure 3. Rich network of thin reddish vessels (arrow) lined with atypical endothelial cells, in the context of breast glandular stroma (hematoxylin eosin staining; magnification, $\mathrm{x} 200$ ).

$\sim 25 \mathrm{~mm}$ in diameter, surrounded by a hyperaemic area, with no other systemic symptoms or abnormal clinical findings (Fig. 1). An ultrasound scan (Esaote MyLab ${ }^{\mathrm{TM}}$ 25; Esaote SpA, Genoa, Italy) of the breast confirmed a circumscribed hypo-anechoic oval nodule ( $22 \mathrm{~mm}$ in diameter) located in

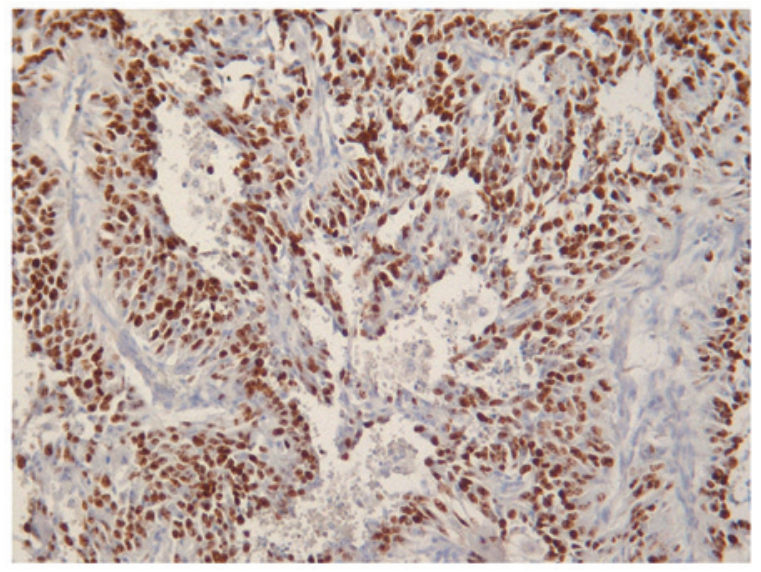

Figure 4. Tumour cell proliferation was high. MIB-1 labelling index, $>80 \%$ (immunoperoxidase + Mayer's haemalum counterstain; magnification, $x 160$ ).

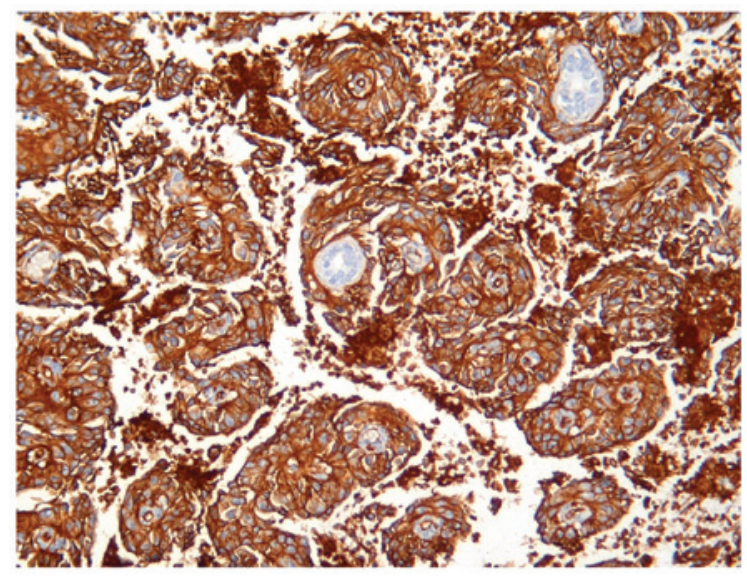

Figure 5. Intense and homogeneous cytoplasmic immunoreactivity for CD31 antibody (immunoperoxidase + Mayer's haemalum counterstain; magnification, $\mathrm{x} 160$ ).

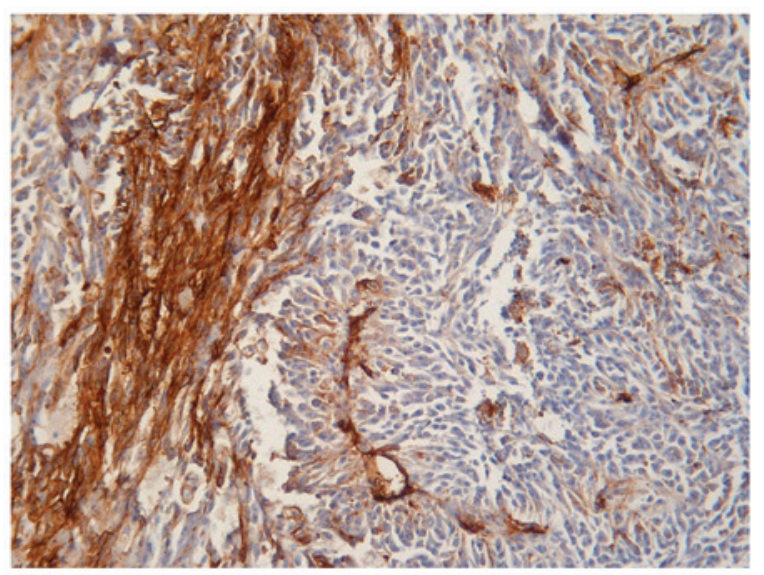

Figure 6. Intense and homogeneous cytoplasmic immunoreactivity for CD34 antibody (immunoperoxidase + Mayer's haemalum counterstain; magnification, x160).

the left upper-inner breast quadrant, with an irregular vascular pattern observed on colour Doppler, and a posterior acoustic increase (Fig. 2). A previous practitioner had considered this finding to be suggestive of an abscess, despite the history 
of neoplasia in the patient. Following fine-needle aspiration cytology, performed by means of a 25 -gauge needle, a mesenchymal neoplasm was suspected; however, these findings were inconclusive and did not allow the confirmation of a diagnosis. Routine laboratory investigations yielded normal results, with the exception of an elevated C-reactive protein level of $1.9 \mathrm{mg} / \mathrm{dl}$ (normal range, $0-0.5 \mathrm{mg} / \mathrm{dl}$ ). Standard thorax radiography did not reveal any parenchymal lesions or pleural effusions. Values for the tumour markers carcinoembryonic antigen, carbohydrate antigen (CA)15-3 and CA125 were in the normal ranges.

Based on these findings, the patient was subjected to a superomedial second-look excision. The tumour was highly vascularised, and the estimated blood loss was $\sim 50 \mathrm{ml}$. An aspirative drain was placed locally. Grossly, the surgical specimen measured $55 \times 28 \times 25 \mathrm{~mm}$ and appeared as a cutaneous, greyish, nodular lesion ( $25 \mathrm{~mm}$ in its greatest diameter).

Histological examination of the specimen was conducted as follows. Formalin-fixed and paraffin-embedded specimens were cut into $4 \mu \mathrm{m}$-thick serial sections and mounted on silane-coated glass slides. Following de-waxing in xylene and re-hydration in graded ethanol, antigen retrieval was performed by heating slides in $0.01 \mathrm{M}$ citrate buffer $(\mathrm{pH}$ 6.0) in a microwave oven for $3 \times 5 \mathrm{~min}$. For the immunohistochemical study, sections were incubated in a moist chamber with $0.1 \% \mathrm{H}_{2} \mathrm{O}_{2}$ in methanol to block intrinsic peroxidase activity, and then with normal sheep serum to prevent nonspecific adherence of serum proteins. Subsequently, the slides were incubated with the following primary antibodies: Mouse monoclonal anti-human vimentin (\#GA630; Clone V9; Dako, Glostrup, Denmark; dilution, 1:100), CD34 (\#GA632; Clone QBEnd 10; Dako; 1:50), CD31 (\#GA610; Clone JC70A; Dako; 1:50) and Ki-67 (\#GA626; Clone MIB-1; Dako; 1:75). The slides were then incubated with sheep anti-mouse IgG antiserum (\#ab6808; Abcam, Cambridge, UK; 1:25) and mouse anti-horseradish peroxidase-antiperoxidase complexes (LSAB 2 System-HRP; \#K0672; Dako; 1:25) for 30 min each at room temperature. For the demonstration of peroxidase activity the sections were incubated in darkness for $10 \mathrm{~min}$ with 3-3'-diaminobenzidine tetra hydrochloride (Sigma-Aldrich, St. Louis, MO, USA). Nuclear counterstaining was conducted with Mayer's haemalum. Negative controls included omission of the primary antiserum and replacement of the primary antiserum with phosphate-buffered saline solution $(\mathrm{pH}$ 7.4) or normal horse serum; in each of these conditions, no staining was evident. Histological sections of capillary haemangiomas/haemangioblastomas were utilized as positive controls for CD34 and CD31.

The microscopic appearance following hematoxylin-eosin routine staining was consistent with recurrent BA. The tumour exhibited a pseudo-papillary architecture constituted by a vascular core surrounded by spindle- or oval-shaped cells, with frequent mitoses, and significant infiltration of the superficial and deep dermis (Fig. 3). An increased rate of neoplastic cell proliferation was revealed by the high MIB-1 LI (>80\%; Fig. 4). On immunostaining, the evident cytoplasmic immunoreactivity for CD31 and CD34 confirmed the endothelial nature of the proliferating cells (Figs. 5 and 6), and the immunopositivity for vimentin further supported the mesenchymal origin. Surgical margins were unaffected and clear.
The patient's in-hospital stay was uneventful, the drainage was removed, and she was discharged on the third postoperative day. No complementary treatment was prescribed. At 25 months post-surgery, the patient was well and free of any clinical local recurrence or distant metastases. In the follow-up period, locoregional ultrasound examination findings were normal, and whole-body scintigraphy and brain-thoracoabdominal computed tomography scans (LightSpeed VCT; GE Healthcare, Fairfield, CT, USA) did not reveal any recurrence or metastatic lesions.

Informed consent for the current report was obtained from the patient.

\section{Discussion}

Angiosarcomas represent $<2 \%$ of all soft tissue sarcomas $(1,5)$. BAs are rare neoplasms that develop from the endothelial lining of the blood vessels (1-3). These tumours are considered as primary in the absence of known risk factors and, with an uncertain aetiology, they affect typically women aged $<40$ years $(1,2)$.

Secondary BAs generally occur in elderly patients subsequent to adjuvant external-beam radiation therapy performed with a breast-conserving surgery, with or without lymphoedema (5). The first case of BA following conservative surgery in association with radiation was reported in 1987 (9). A number of reports indicate that, following the initial surgical and radiation therapies, the interval of latency for the onset of an angiosarcoma is 3-12 years $(1,5,6)$. These tumours are infiltrative, non-capsulated, cutaneous, soft-tissue sarcomas (2). Their incidence has been rising over the past 30 years (3). Histologically, they are characterised by high cellularity, consisting of pleomorphic endothelial cells, spindle-shaped, rounded or oval-shaped, arranged in loose or cohesive clusters or sheets, or sometimes as single cells with a scanty pale blue cytoplasm (6). BAs are classified according to Donnel's Classification (10). Well-differentiated tumours include open anastomosing vascular sinusoids, with a single layer of abnormal endothelial cells that exhibit hyperchromatic nuclei and few mitoses (2). In increasingly aggressive disease, classified as a moderately differentiated angiosarcoma, the vascular channels are less clearly defined, and are associated with small foci of spindle-shaped cells, which are multilayered or arranged in pseudopapillary structures with several mitoses (6). The poorly differentiated variety exhibits malignant endothelial cells organised in continuous sheets, generally with epithelioid features, blood lakes and necrosis (2).

Immunohistochemistry is useful to confirm the diagnosis of BA $(2,6,10)$. Typically, the tumors are positive for endothelial markers, including CD31, CD34, von Willebrand factor and vascular endothelial growth factor (2). The pathological differential diagnosis of this tumour includes phyllodes sarcoma, stromal sarcoma, benign hemangioma, myoepithelioma fibromatosis, fibrosarcoma, liposarcoma, metaplastic carcinoma, squamous cell carcinoma with sarcomatoid features and high-grade mammary carcinoma (3). The diagnosis of secondary BA is predominantly clinical at first observation, yet its appearance is highly variable in form $(5,6)$. In the literature, these tumours have been described as a palpable masses, which may be painful or painless, or as an erythematous area 
or even as a lipoma $(1,3,11)$. A fine-needle aspiration biopsy is highly recommended $(2,6)$.

A wide-margin surgical excision represents the treatment of choice $(2,5,12)$; supplementary radiotherapy boost is typically avoided in cases of radiation-associated BA (2). Generally, BAs are localised and the tumour recurrences are most frequent within 1 year from surgical treatment $(5,12)$. Affected or unclear surgical margins and large tumour size are considered poor prognostic factors $(2,5)$. Due to the increase in complementary radiotherapy following breast conservative treatment, great attention to skin alterations is recommended during the follow-up period in order to allow the early detection of secondary lesions $(1,5)$. Biopsies must be encouraged, and radiological results should be analysed by breast surgery specialists.

In conclusion, the current study reports a case of BA in an elderly woman which recurred twice, 11 years after a breast-conserving surgery with adjuvant radiotherapy, and 5 years after the patient's first angiosarcoma excision. To the best of our knowledge, this has not previously been reported in the literature. Clinicians should be aware of this unusual occurrence and of any possible misdiagnosis.

\section{Acknowledgements}

The authors wish to thank Dr Giuseppe Campisi, a member of the surgical team at the University Hospital of Messina.

\section{References}

1. Vorburger SA, Xing Y, Hunt KK, Lakin GE, Benjamin RS, Feig BW, Pisters PW, Ballo MT, Chen L, Trent J III, et al: Angiosarcoma of the breast. Cancer 104: 2682-2688, 2005.
2. Young RJ, Brown NJ, Reed MW, Hughes D and Woll PJ: Angiosarcoma. Lancet Oncol 11: 983-991, 2010.

3. Scow JS, Reynolds CA, Degnim AC, Petersen IA, Jakub JW and Boughey JC: Primary and secondary angiosarcoma of the breast: The mayo clinic experience. J Surg Oncol 101: 401-407, 2010.

4. Zelek L, Llombart-Cussac A, Terrier P, Pivot X, Guinebretiere JM, Le Pechoux C, Tursz T, Rochard F, Spielmann $\mathrm{M}$ and Le Cesne A: Prognostic factors in primary breast sarcomas: A series of patients with long-term follow-up. J Clin Oncol 21: 2583-2588, 2003.

5. Monroe AT, Feigenberg SJ and Mendenhall NP: Angiosarcoma after breast-conserving therapy. Cancer 97: 1832-1840, 2003.

6. Gherardi G, Rossi S, Perrone S and Scanni A: Angiosarcoma after breast-conserving therapy: Fine-needle aspiration biopsy, immunocytochemistry and clinicopathologic correlates. Cancer 105: 145-151, 2005.

7. Madden JL: Modified radical mastectomy. Surg Gynecol Obstet 121: 1221-1230, 1965

8. Wildiers H, Kunkler I, Biganzoli L, Fracheboud J, Vlastos G, Bernard-Marty C, Hurria A, Extermann M, Girre V, Brain E, et al; International Society of Geriatric Oncology: Management of breast cancer in elderly individuals: recommendations of the International Society of Geriatric Oncology. Lancet Oncol 8: 1101-1115, 2007.

9. Body G, Sauvanet E, Calais G, Fignon A, Fetissof F and Lansac J: Cutaneous angiosarcoma of the breast following surgery and irradiation of breast adenocarcinoma. J Gynecol Obstet Biol Reprod (Paris) 16: 479-483, 1987.

10. Donnell RM, Rosen PP, Lieberman PH, Kaufman RJ, Kay S, Braun DW Jr and Kinne DW: Angiosarcoma and other vascular tumors of the breast. Am J Surg Pathol 5: 629-642, 1981.

11. Meroni S, Moscovici O, Menna S, Renne G, Sosnovskikh I, Rossi V and Cassano E: Ultrasound challenge: Secondary breast angiosarcoma mimicking lipoma. Breast J 19: 437-438, 2013.

12. Biswas T, Tang P, Muhs A and Ling M: Angiosarcoma of the breast: A rare clinicopathological entity. Am J Clin Oncol 32: $582-586,2009$. 